\title{
Statistical analyses of cyclic and starlike hierarchical dominances in directed graphs
}

\author{
György Szabó $\odot^{*}$ and István Borsos ${ }^{\dagger}$ \\ Institute of Technical Physics and Materials Science, Centre for Energy Research, Hungarian Academy of Sciences, \\ P.O. Box 49, H-1525 Budapest, Hungary \\ Borbála Leitner \\ Department of Physics, Eövös University, Pámány Péter sétány 1A, H-1117 Budapest, Hungary
}

(Received 20 March 2019; published 3 September 2019)

\begin{abstract}
The mathematical framework of matrix decomposition implies the possibility to perform statistical analyses of directed graphs focused on the distributions of the independent cyclic and starlike hierarchical components. In this approach the weighted directed graphs with $n$ nodes are built up as a linear combination of starlike graphs with $n$ outgoing edges and a suitable set of three-edge cyclic subgraphs. The applicability of this approach is illustrated by quantifying several general features: e.g., ratio of the cyclic and hierarchical components and asymmetry in the hierarchical components. The applicability of these methods is illustrated by considering the averages over random directed graphs and comparing these values with those characterizing simple directed graphs of tournaments.
\end{abstract}

DOI: 10.1103/PhysRevE.100.032301

\section{INTRODUCTION}

Graph theory and networks are widely used to quantify the structures of social, biological, neural, and many other complex systems [1-6]. These graphs are composed of nodes (points with labels $i=1,2, \ldots, n$ ) and links (edges) connecting two nodes. The structural properties of these objects are described by a large number of concepts and quantities $[4,7,8]$, for example, the numbers of nodes and edges, the degree (number of edges) for nodes, the degree distribution, clustering coefficients, etc. The adequate description of the more realistic systems has required the generalization of the original simple objects and the introduction of more sophisticated quantities. As a result further quantities are introduced and studied. Some of these concepts are characteristic of the global system (e.g., spanning trees, Hamiltonian loops, centrality, diameter, and hierarchy) while others are related to local features, such as the cliques or communities, overlapping communities [9], motifs, and directed network modules [10].

The analyses of graphs and matrices are strongly related to each other via the adjacency matrix $\mathbf{A}$ with elements $A_{i j}$ quantifying the connection between the nodes $i$ and $j[1,11]$. In game theory [12-14] and evolutionary game theory [15-17] an $n \times n$ payoff matrix $\mathbf{A}$ defines the interaction between two equivalent players having $n$ options and they choose one of them independent of each other. Recently it was found that the game-theoretical interactions can be composed of four types of elementary interactions defined by matrices possessing different symmetries [18,19]. In this approach the matrix is considered as an $n^{2}$-dimensional vector which can be built

\footnotetext{
*szabo@mfa.kfki.hu

†borsos@mfa.kfki.hu

†hudak64@gmail.com
}

up as a suitable linear combination of $n^{2}$ orthogonal basis matrices. The straightforward adoption of the Cartesian basis vectors defines a set of orthogonal basis matrices containing a single 1 among zeros. Instead of the latter basis matrices, however, one can introduce another complete set of basis matrices which reflects the inherent symmetries of the matrices. Such a set of basis matrices can be constructed from dyadic products of $n$-dimensional basis vectors if one of them is the all-one vector [18]. For this construction four types of elementary interactions can be distinguished in the game theory, namely, games with self- and cross-dependent payoffs, coordination-type interactions, and combinations of elementary cyclic dominance. The antisymmetric part of the self- and cross-dependent components can cause social dilemmas in the potential games [20] while the presence of cyclic components prohibits the existence of potential.

Now we adapt the above mathematical framework to the analyses of directed weighted graphs possessing antisymmetric adjacency matrices $\left(\mathbf{A}^{T}=-\mathbf{A}\right)$. These graphs can have a single weighted and directed edge between any pair of nodes. This limitation simplifies the analysis significantly because here we have only two (orthogonal) types of elementary interactions to be considered separately [21]. The antisymmetric part of the self- and cross-dependent terms represents the starlike hierarchical components while the rest of matrix $\mathbf{A}$ defines the cyclic components. The latter components satisfy a criterion, namely, that the sum of the matrix elements is zero in each row and column. It means in the corresponding graphs that the total weights of incoming and outgoing edges are equal for each node, and these graphs can be built up from three-edge directed loops representing voluntary rockpaper-scissors games [21]. In this approach the $i$ th elementary starlike hierarchical component corresponds to a directed graph possessing directed edges from the node $i$ to all others with a unit strength. 
The general mathematical framework of this decomposition is briefly surveyed in Sec. II (more complete discussions are given in $[18,19,21])$. The applicability of this approach is demonstrated by introducing several quantities and determining their average values over the whole set of complete directed random graphs. The score table of a round robin tournament can also be related to a directed graph $[3,22,23]$. Using these tools we compare the score tables of women's handball championships obtained for the first and second classes exhibiting different features.

\section{MATHEMATICAL BACKGROUND}

We consider simple directed graphs $\mathcal{G}$ with $n$ nodes labeled by $i=1,2, \ldots, n[1,3]$. The nodes $i$ and $j(1 \leqslant i \neq j \leqslant n)$ are connected to each other by a single directed edge with a weight factor $A_{i j}$ defining the elements of the antisymmetric adjacency matrix $\left(\mathbf{A}=-\mathbf{A}^{T}\right.$ or $\left.A_{i j}=-A_{j i}\right)$. More precisely, $A_{i j}>0$ quantifies the weight of a directed edge from node $i$ to $j$ while $A_{i j}=A_{j i}=0$ indicates the absence of a directed link between the nodes $i$ and $j$.

As mentioned in the Introduction, a matrix $\mathbf{A}$ can be built up as a linear combination of $n^{2}$ (independent) elementary basis matrices. It is known that the dyadic products of the $n$-dimensional basis vectors can serve as a suitable orthogonal set of $n \times n$ basis matrices. If these dyads are constructed from the Cartesian basis vectors then we get basis matrices containing zeros and a single 1 . Instead of it, we can choose any other rotated coordinate systems. For the classification of pair interactions in game theory a suitable decomposition is based on the dyadic products of such a rotated $n$-dimensional coordinate system where the all-one vector plays a distinguished role and we can distinguish four types of interactions, named as games with self- and cross-dependent payoffs, coordination, and games with cyclic dominance $[18,19]$. Accordingly, the dyadic product of the all-one vector with itself defines the all-one matrix representing an irrelevant term in the matrix games. Independent of the choice of the other basis vectors the dyadic products of the all-one vector with the suitable orthogonal vector components define games with self- and cross-dependent payoffs. For the self-dependent (cross-dependent) games the matrix elements are equivalent in the rows (columns). The antisymmetric part (denoted as $\mathbf{A}^{(\mathrm{h})}$ ) of the latter two types of interactions can be defined by $(n-1)$ parameters and these components are responsible for the appearance of social dilemmas in the potential games [20]. The rest of the dyadic products can also be separated into the sum of symmetric and antisymmetric matrices. The symmetric parts quantify the strengths of coordination (or Ising-like) interactions between different strategy pairs while the antisymmetric part (denoted as $\mathbf{A}^{(\mathrm{c})}$ ) describes the contributions of cyclic dominance [21] preventing the existence of potential and thermodynamical behavior.

In light of the above results the antisymmetric adjacency matrix $\mathbf{A}$ can be considered as a $D$-dimensional $(D=n(n-$ 1)/2) vector with components parametrizing the directed graph. These matrices can be given as a sum of the hierarchical and cyclic components:

$$
\mathbf{A}=\mathbf{A}^{(\mathrm{h})}+\mathbf{A}^{(\mathrm{c})} \text {. }
$$

The graph representation of this relationship is discussed in [21], where it is shown how a single directed edge from node $i$ to $j$ is composed of two starlike hierarchical elementary components and many other cyclic components. More precisely, the first hierarchical elementary component $(\mathcal{H}(i))$ contains $(n-1)$ equivalent outgoing edges from site $i$ while the second one $(-\mathcal{H}(j))$ has only $(n-1)$ equivalent incoming edges at site $j$. The unnecessary edges are eliminated by adding $(n-2)$ three-edge directed loops $[i \rightarrow j \rightarrow k \rightarrow i]$ where $k$ runs over the rest of the sites.

The hierarchical component $\mathbf{A}^{(\mathrm{h})}$ is the linear combination of $n$ elementary matrices,

$$
\mathbf{A}^{(\mathrm{h})}=\frac{1}{n} \sum_{i} h(i) \mathbf{H}(i),
$$

where the values of $h(i)$ satisfy the following condition:

$$
\sum_{i} h(i)=0 \text {. }
$$

This notation expresses the equivalence of nodes and the fact that we have only $(n-1)$ independent elementary $\mathbf{H}(i)$ matrices. The matrix $\mathbf{H}(i)$ is the adjacency matrix of a starlike directed hierarchical graph $\mathcal{H}(i)$ mentioned above. For example,

$$
\mathbf{H}(1)=\left(\begin{array}{cccc}
0 & 1 & \ldots & 1 \\
-1 & 0 & \ldots & 0 \\
\vdots & \vdots & \ddots & \vdots \\
-1 & 0 & \ldots & 0
\end{array}\right)
$$

that can be expressed by the mentioned dyadic products as

$$
\mathbf{H}(1)=\left(\begin{array}{cccc}
1 & 1 & \ldots & 1 \\
0 & 0 & \ldots & 0 \\
\vdots & \vdots & \ddots & \vdots \\
0 & 0 & \ldots & 0
\end{array}\right)-\left(\begin{array}{cccc}
1 & 0 & \ldots & 0 \\
1 & 0 & \ldots & 0 \\
\vdots & \vdots & \ddots & \vdots \\
1 & 0 & \ldots & 0
\end{array}\right) .
$$

As indicated this matrix is deduced from two dyadic products created from the all-one vector and the first Cartesian basis vector [18]. $\mathbf{H}(i)$ can be obtained from $\mathbf{H}(1)$ by exchanging the first and $i$ th rows and columns simultaneously. Notice that $\sum_{i} \mathbf{H}(i)=0$. In this approach the values of the $h(i)$ coefficient are given by the sums of matrix elements in the $i$ th row, that is,

$$
h(i)=\sum_{j} A_{i j}
$$

For simple directed graphs $h(i)$ is the difference of outgoing and incoming edges for node $i$ and these quantities are generally used to rank the hierarchy of nodes.

Before discussing the cyclic component $\mathbf{A}^{(\mathrm{c})}$ we adopt the concepts of scalar product and orthogonality for matrices. Accordingly, the scalar product of the matrices $\mathbf{A}$ and $\mathbf{B}$ is defined as

$$
\mathbf{A} \cdot \mathbf{B}=\sum_{i, j} A_{i j} B_{i j}
$$

and these two matrices are orthogonal to each other if their scalar product is zero. 
It is worth mentioning that the elementary components $\mathbf{H}(i)$ are not orthogonal to each other. More precisely, $\mathbf{H}(i)$. $\mathbf{H}(j)=-2$ if $i \neq j$ and $\mathbf{H}(i) \cdot \mathbf{H}(i)=2(n-1)$. Furthermore, if $\mathbf{A}=\mathbf{H}(1)$ then $h(1)=n-1$ and $h(i)=-1$ if $i>1$.

The orthogonality of the hierarchical and cyclic components $\left(\mathbf{A}^{(\mathrm{c})} \cdot \mathbf{A}^{(\mathrm{h})}=0\right.$ ) dictates that the (weighted) sum of the edges is zero for each node $i$ of the cyclic component, that is, $\sum_{j} A_{i j}^{(\mathrm{c})}=0$. Contrary to the treelike hierarchical elementary components, the cyclic components $\mathbf{A}^{(\mathrm{c})}$ include directed loops. The reader can easily check that any directed loops (as well as their linear combinations) can be built up from suitable three-edge directed loops [21]. Accordingly, we can introduce the elementary cyclic components $\mathbf{C}(i, j, k)$ which are the adjacency matrices of the three-edge directed graphs with edges from $i$ to $j, j$ to $k$, and $k$ to $i$. For example,

$$
\mathbf{C}(1,2,3)=\left(\begin{array}{rrrrrr}
0 & 1 & -1 & 0 & \ldots & 0 \\
-1 & 0 & 1 & 0 & \ldots & 0 \\
1 & -1 & 0 & 0 & \ldots & 0 \\
0 & 0 & 0 & 0 & \ldots & 0 \\
\vdots & \vdots & \vdots & \vdots & \ddots & \vdots \\
0 & 0 & 0 & 0 & \ldots & 0
\end{array}\right)
$$

and the matrices $\mathbf{C}(i, j, k)$ can be obtained from $\mathbf{C}(1,2,3)$ by exchanging suitable rows and columns $(1 \leftrightarrow i, 2 \leftrightarrow j$, and $3 \leftrightarrow k$ ). These sparse matrices contain one +1 and -1 in the corresponding rows and columns. The cyclic permutation of indices leaves the matrix unchanged while the simultaneous reverse of edge directions yields $\mathbf{C}(i, j, k)=-\mathbf{C}(j, i, k)$. The total number of these cyclic components, $\left(\begin{array}{l}n \\ 3\end{array}\right)$, exceeds the number of independent components. It is found, however, that one can easily choose a suitable (complete and independent) subset of $\mathbf{C}(i, j, k)$ s to express the cyclic component unambiguously as

$$
\mathbf{A}^{(\mathrm{c})}=\sum_{\substack{j \\ j>1}} \sum_{\substack{k \\ k>j}} c(1, j, k) \mathbf{C}(1, j, k),
$$

where the three-edge loops are limited to those involving the node $i=1$ [21]. It is noteworthy that $c(1, j, k)=A_{j k}^{(c)}$ for $1<$ $j<k \leqslant n$ because the other elementary cyclic components give zero contributions to the matrix element $A_{j k}^{(c)}$. Evidently, there are $(n-1)$ additional suitable independent subsets with $\left(\begin{array}{c}n-1 \\ 2\end{array}\right)$ elementary components where the distinguished role of the node $i=1$ is replaced by another node.

The hierarchical and cyclic components of $\mathbf{A}$ are orthogonal, that is, $\mathbf{A}^{(\mathrm{h})} \cdot \mathbf{A}^{(\mathrm{c})}=0$, due to the orthogonality between their elementary components, namely, $\mathbf{H}\left(i^{\prime}\right) \cdot \mathbf{C}(i, j, k)=0$ for all possible values of indices. This feature allows us to measure the ratios of the hierarchical $\mathbf{H}^{(\mathrm{h})}$ and cyclic $\mathbf{H}^{(\mathrm{c})}$ components with their scalar projection onto A. Namely, the portion $\Lambda$ of the hierarchical components of $\mathbf{A}$ can be quantified as

$$
\Lambda=\frac{\mathbf{A}^{(\mathrm{h})} \cdot \mathbf{A}}{\mathbf{A} \cdot \mathbf{A}}=\frac{\mathbf{A}^{(\mathrm{h})} \cdot \mathbf{A}^{(\mathrm{h})}}{\mathbf{A} \cdot \mathbf{A}} \geqslant 0 .
$$

Similarly, the portion $\Theta$ of cyclic components,

$$
\Theta=\frac{\mathbf{A}^{(\mathrm{c})} \cdot \mathbf{A}}{\mathbf{A} \cdot \mathbf{A}}=\frac{\mathbf{A}^{(\mathrm{c})} \cdot \mathbf{A}^{(\mathrm{c})}}{\mathbf{A} \cdot \mathbf{A}} \geqslant 0 .
$$

Due to the orthogonality relation, these quantities satisfy the condition $\Lambda+\Theta=1$.

Finally we underline that in this approach a directed graph is defined by $(n-1)$ hierarchical coefficients $h(i)$ and $\left(\begin{array}{c}n-1 \\ 2\end{array}\right)$ cyclic coefficients $c(1, j, k)$ instead of the $\left(\begin{array}{c}n \\ 2\end{array}\right)$ independent $A_{i j}$ matrix elements. Noteworthy is that the portion of the number of independent hierarchical components decreases with $n$ as $2 / n$.

\section{ROUND ROBIN TOURNAMENTS}

The round robin tournaments represent a well-studied class of directed graphs [3,22]. In this tournament $n$ participants compete with all others (in pairs) to win points from each other. The score table summarizes the results for all pairs. At the end of competition the ranks of players are determined by the points $[h(i)$ in Eq. (6)] they collected. If the pair competitions are similar to a zero-sum game then the score table is equivalent to an antisymmetric matrix A. Henceforth our attention is focused on simple tournaments where the winners of pair interactions receive +1 , the losers -1 , and both get zero for a draw.

In tournaments there are two extreme situations, represented by $\mathbf{A}=\mathbf{H}(i)$ or $\mathbf{A}=-\mathbf{H}(i)$. In the first (winner dominated) case the $i$ th player overcomes all others who draw against each other (or collect zero points from others). The opposite case represents a loser dominated competition where the $i$ th participant is beaten by every player while their competition yields zero point for each. The quantity $\Psi$,

$$
\Psi=\frac{1}{n-2} \frac{\sum_{i} h^{3}(i)}{\sum_{i} h^{2}(i)},
$$

measures the asymmetry of hierarchy in real systems. For winner (loser) dominated tournaments $\Psi=1(-1)$. Simple analytical calculations justify that the modification of the score table of $\mathbf{A}=\mathbf{H}(i)$ for a single pair interaction reduces $\Psi$ from its maximum value. Evidently, if $\mathbf{A}=\mathbf{H}(1)-\mathbf{H}(n)$, then $\Psi=0$. In general, $\Psi \in[-1,1]$.

\section{A. National handball championship}

To illustrate the quantitative determination of $\Lambda, \Theta$, and $\Psi$ in real directed graphs we now investigate the women's handball championships both in the autumn and spring seasons of 2016-2017 in Hungary. In the national championship there are $n=14$ teams in the first class. In every year at the end of these championships the worst two teams of the first class are replaced by the winners of two second class championships played by $n=14$ teams of the east and west parts of the country. The worst teams in the second classes are also replaced by the winners of the third classes, etc. These rules yield different evolutionary processes for the first and second classes in the whole national championship. Additional differences between the classes are caused by the sponsorships favoring the best teams.

In the knowledge of the results of matches we have determined the simplified score tables (with $A_{i j}=+1,-1$, or 0 ) and evaluated the quantities introduced above. The frequency of a draw is very rare in handball championships. In this national championship the first class is dominated by two 
teams; one of them won the continental championships several times in recent years. More precisely, the winner won all matches in both seasons while the last team won four matches in the two seasons. For these reasons we expected a relevant asymmetry in the value of $\Psi$. The numerical results are $\Psi=$ 0.26 and $0.15 ; \Lambda=0.53$ and 0.56 in the autumn and spring seasons. In contrast, in the four tournaments of the second classes the values of $\Lambda$ varied between 0.30 and 0.53 while the antisymmetry parameters of the hierarchy $(\Psi)$ were lower (typically, close to zero) than what we found in the first class. In one of these second class tournaments, however, there was a team beaten by all others, and this fact is clearly indicated by the negative value of $\Psi(\Psi=-0.20)$.

In the next section the above numerical results are compared with average values obtained over a suitable set of random directed graphs.

\section{B. Matching pennies tournaments}

In order to have a reference basis now we study a matching pennies tournament when the competitors can get (or lose) a unit payoff from their co-player at random. For this competition the score table is an antisymmetric random matrix with elements $A_{i j}=-A_{j i}=1$ or -1 . In the corresponding complete simple graph the edge directions are chosen at random.

First the applicability of the above quantities is tested by performing numerical simulations for $n=14$. More precisely, a huge number $\left(N=10^{10}\right)$ of score tables are generated at random and we have determined the average values of the quantities introduced above. According to these numerical analyses the average values are $\langle\Lambda\rangle=0.142858(1)$ and $\langle\Theta\rangle=0.857142(1)$. Evidently, the average value of $\Psi$ is zero because both $\mathbf{A}$ and $\mathbf{- A}$ belong to the set of matrices investigated.

The effects of $n$ on the average values of $\Lambda$ and $\Theta$ are quantified by additional numerical simulations performed for several values of $n$. The results are consistent with the conjecture: $\langle\Lambda\rangle=2 / n$ and $\langle\Theta\rangle=1-2 / n$. It means that the average portion of the hierarchical and cyclic components is determined by the ratio of their dimensions. As mentioned above a similar ratio is found when a single directed edge is decomposed into two starlike hierarchical and $(n-2)$ cyclic components [21]. This observation indicates that the elementary basis matrices are equivalent and give identical contributions to the average portions $\Lambda$ and $\Theta$.

Subsequently we have studied what happens if a portion $\rho$ of edges is removed. This modification describes tournaments where a portion $\rho$ of pair interactions end with a draw. If $\rho=$ $1 / 3$ then the round robin tournament is based on a rock-paperscissors game. Interestingly, the removal of edges does not modify the average values of $\Lambda$ and $\Psi$.

The possible values of the asymmetry of hierarchy are limited to a subset of rational numbers. In order to derive a smooth probability distribution as a function of $\Psi$ the whole region $[-1,+1]$ is divided into $k=37$ segments with equivalent extension and the average probabilities are determined over all segments. This method is also applied when we quantified the $\Psi$ dependence of the average values of $\Lambda$. The same statistical analyses are repeated when a portion $\rho$ of directed

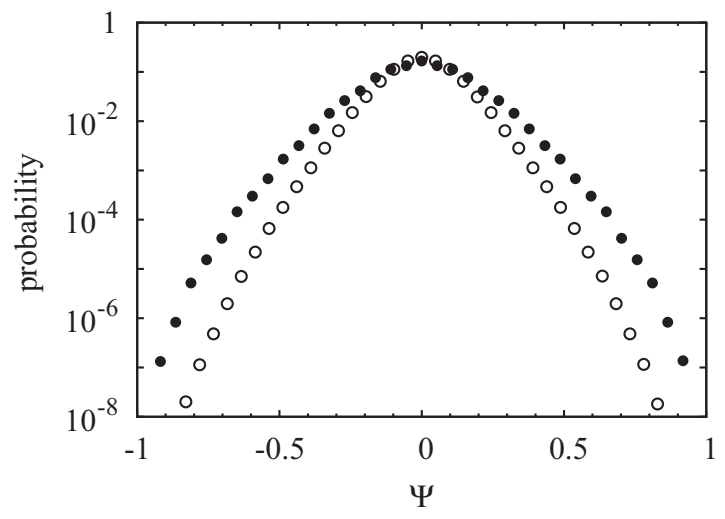

FIG. 1. Smoothed probability distribution of finding a directed graph with a hierarchical asymmetry $\Psi$ for $n=14$ if $\rho=0$ (solid circles) and $\rho=0.5$ (open circles).

edges are removed. Figure 1 compares the results obtained for the complete and half-filled random directed graphs. The results illustrate that this smoothed probability distribution is symmetric and goes to its minimum at the boundaries. The irregularities are related to the statistical errors and occasional situations when $\Psi$ is located on a boundary separating two neighboring segments. The latter discrepancy is reduced for $k=37$. The numerical results in Fig. 1 show clearly that the random elimination of half of the edges decreases the probability of reaching high $|\Psi|$; that is, the smoothed probability distribution becomes narrower while its symmetry is preserved.

The parameters $\Lambda$ and $\Psi$ characterize directed graphs. Thus, each graph can be represented by a point on the $\Psi-\Lambda$ plane. In Fig. 2 solid diamonds and open boxes illustrate the location of these parameters for the score sheets discussed in the previous section. The characteristics of these score sheets are contrasted with the average values obtained by

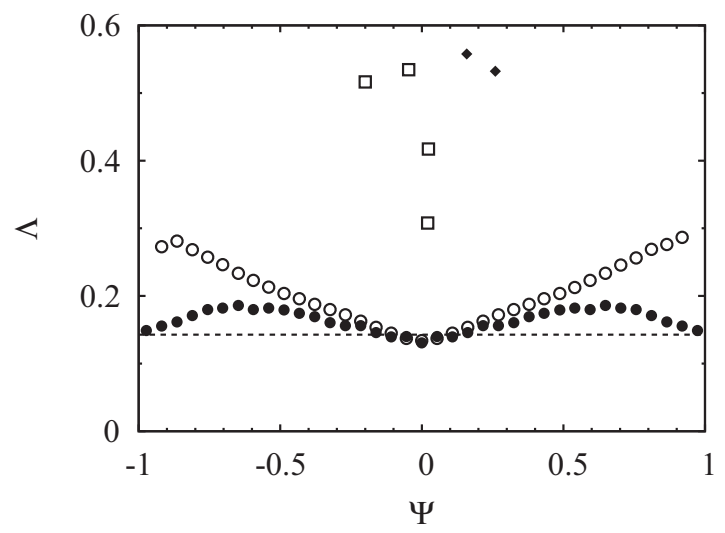

FIG. 2. Location of real score sheets on the $\Psi-\Lambda$ plane. Solid diamonds and open boxes illustrate these features of the National Women's Handball Championship in the autumn and spring seasons of 2016-2017 in Hungary for the first and second classes. For $n=14$ the $\Psi$ dependence of the average values of the portion of hierarchical components is shown by solid and open circles for $\rho=0$ and $\rho=$ 0.5 . The dashed line shows the average value of $\Lambda$ over the whole set of directed random graphs. 
averaging over the whole set of random directed graphs. Additionally we have determined the $\Psi$ dependence of the average values of $\Lambda$ over suitable subsets of graphs. The latter results indicate that the $\Psi$ dependence of $\Lambda$ is not relevant for dense graphs. In contrast, for $\rho=0.5$ the portion of the hierarchical components increases linearly with $|\Psi|$.

The most remarkable message of Fig. 2 is the high values of $\Lambda$ in real networks (tournaments) that reflect clearly the relevance of differences in the quality of teams in the national championships. Additionally, the difference between the first and second class championships may be related to the evolutionary processes affecting the characteristics of the score tables. Thus, in the second classes both the best and worst teams are replaced by others, and these symmetric changes prevent relevant deviations from the value $\Psi=0$. In contrast, in the first class the best team remains in the same class, and, due to the sponsors, it can improve the motivation of team members and also its quality by inviting better players.

\section{SUMMARY}

The present study is devoted to demonstrating the applicability of some concepts of the matrix decomposition in the field of network analysis. For simplicity, our analysis is now restricted to the antisymmetric matrices and the corresponding simple directed graphs where only two types of elementary orthogonal components can be distinguished. We have introduced three quantities characterizing the macroscopic features of directed graphs. It is shown that the portion of the hierarchical $\Lambda$ and cyclic $\Theta=1-\Lambda$ components can be measured by their scalar projection onto the antisymmetric adjacency matrix. It is also illustrated that the possible antisymmetry of the hierarchical components can also be quantified by a parameter $\Psi$ varying between -1 and +1 .

We emphasize that the above quantities are mathematically well defined and utilize the concept of orthogonality and symmetry. The elementary motifs of the hierarchical components are represented by starlike directed graphs involving all nodes. There are $n$ equivalent elementary starlike hierarchical components while the number of independent components is only $(n-1)$. The reduction of the number of independent components is compensated by the mathematical formulas preserving the equivalence of nodes. Namely, all these components appear with a weight factor $h(i) / n$ in the formulas while the sum of these coefficients is zero.

The entanglement of the cyclic components is more complicated because there exist $\left(\begin{array}{l}n \\ 3\end{array}\right)$ three-edge elementary cyclic components while the number of independent components is only $\left(\begin{array}{c}n-1 \\ 2\end{array}\right)$. Contrary to the elementary hierarchical components, which refer to global features of the networks, the elementary cyclic components involve only three nodes; thus, they quantify local characteristics. It is found that the cyclic components can be built up unambiguously from those threeedge elementary cyclic graphs which involve a prescribed node. Consequently, there are $n$ equivalent sets of three-edge cyclic components which can be used to build up any cyclic components. Evidently, one can find other ways that the cyclic components can be expressed.

To exemplify the applicability of the quantities $\Lambda$ and $\Psi$ in the network analysis we have studied numerically some tournaments. These characteristic parameters have quantified the differences which may occur in many national championships where first, second, and third classes are distinguished. Additionally, these results justify that the corresponding values deviate significantly from the average values obtained by averaging over random directed graphs if some portion of edges is removed.

When determining numerically the average values of $\Lambda$ for several values of $n$, we faced a general feature. These numerical results are consistent with a simple conjecture: $\langle\Lambda\rangle=2 / n$, namely, the average portions of the hierarchical and cyclic components are proportional to their dimensions defining the independent number of parameters in the corresponding subspaces. A similar value was reported previously when a single directed edge is built up from two hierarchical components and $(n-2)$ three-edge directed loops. These results can serve as a reference basis for the quantitative characterization and comparison of directed networks that are generally formed by an evolutionary process. Finally we emphasize that the analytical justification of this conjecture is a promising challenge.

\section{ACKNOWLEDGMENT}

This work was supported by the Hungarian National Research Fund (Grant No. OTKA TK-120785).
[1] B. Bollobás, Modern Graph Theory (Springer, New York, 1998).

[2] R. Albert and A.-L. Barabási, Rev. Mod. Phys. 74, 47 (2002).

[3] J. Bang-Jensen and G. Z. Gutin, Digraphs: Theory, Algorithms and Applications (Springer-Verlag, London, 2009).

[4] S. Fortunato, Phys. Rep. 486, 75 (2010).

[5] A.-L. Barabási, Network Science (Cambridge University Press, Cambridge, UK, 2016).

[6] A. Zeng, Z. Shen, J. Zhou, J. Wu, Y. Fan, Y. Wang, and E. H. Stanley, Phys. Rep. 714-715, 1 (2017).

[7] M. Girvan and M. E. J. Newman, Proc. Natl. Acad. Sci. USA 99, 7821 (2002).

[8] M. E. J. Newman, SIAM Rev. 45, 167 (2003).
[9] G. Palla, I. Derényi, I. Farkas, and T. Vicsek, Nature (London) 435, 814 (2005).

[10] G. Palla, I. J. Farkas, P. Pollner, I. Derényi, and T. Vicsek, New J. Phys. 9, 186 (2007).

[11] R. B. Bapat, Graphs and Matrices (Springer-Verlag, London, 2014).

[12] J. von Neumann and O. Morgenstern, Theory of Games and Economic Behaviour (Princeton University Press, Princeton, NJ, 1944).

[13] A. Rapoport and A. M. Chammah, Prisoner's Dilemma (University of Michigan Press, Ann Arbor, MI, 1965).

[14] K. Sigmund, The Calculus of Selfishness (Princeton University Press, Princeton, NJ, 2010). 
[15] M. A. Nowak, Evolutionary Dynamics (Harvard University Press, Cambridge, MA, 2006).

[16] G. Szabó and G. Fáth, Phys. Rep. 446, 97 (2007).

[17] B. Allen and M. A. Nowak, EMS Surv. Math. Sci. 1, 113 (2014).

[18] G. Szabó, K. S. Bodó, B. Allen, and M. A. Nowak, Phys. Rev. E 92, 022820 (2015).
[19] G. Szabó and I. Borsos, Phys. Rep. 624, 1 (2016).

[20] G. Szabó and G. Bunth, Phys. Rev. E 97, 012305 (2018).

[21] G. Szabó, K. S. Bodó, and K. A. Samani, Phys. Rev. E 95, 012320 (2017).

[22] H. G. Landau, Bull. Math. Biophys. 15, 143 (1953).

[23] H. A. David, Rev. Int. Stat. Inst. 39, 137 (1971). 